\title{
Investigación como estrategia pedagógica para el desarrollo del género lirico en la escuela
}

\section{Research as a pedagogical strategy for the development of the lyrical genre in school}

DOI: http://dx.doi.org/10.17981/cultedusoc.9.3.2018.36

Artículo de investigación. Fecha de recepción: 15/06/2018. Fecha de aceptación: 27/11/2018

\author{
Maribel Dávila-Mejía; Yanice Arrieta-González; Fredy Fernández-De González; \\ María Acosta-Carvajal; María Alfaro-Baldovino; Félix Gutiérrez-Rodero; \\ Enrique Arrieta-López y Anneth Acuña-Morales ${ }^{1}$ \\ IED Antonio Bruges Carmona, sede Principal (Colombia) \\ maridamej@hotmail.com
}

Para citar este artículo:

Dávila-Mejía, M., Arrieta-González, Y., Fernández-De González, F., Acosta-Carvajal, M., Alfaro-Baldovino, M., Gutiérrez-Rodero, F., Arrieta-López, F. y Acuña-Morales, A. (2018). Investigación como estrategia pedagógica para el desarrollo del género lirico en la escuela. Cultura. Educación y Sociedad 9(3), 317-324. DOI: http://dx.doi.org/10.17981/cultedusoc.9.3.2018.36

\section{Resumen}

La investigación como estrategia pedagógica (IEP) es una metodología educativa que permite la integración de procesos investigativos al aula de clase, en este estudio contribuirá al conocimiento del género lírico aportando al desarrollo de habilidades como la comprensión lectora y los procesos cognitivos superiores de los estudiantes. El propósito del estudio implementar la investigación como estrategia pedagógica para el desarrollo de competencias lectoras mediante el género lirico. La metodología se orientó bajo el enfoque cualitativo, utilizando el tipo de investigación acción, desde un diseño descriptivo, exploratorio. Para la recolección de datos se utilizaron técnicas como; análisis documental. La población estuvo conformada por cincuenta (50) estudiantes de la IED Antonio Bruges Carmona del Municipio de Santa Ana Magdalena, para esto se realizó un muestreo no probabilístico de tipo intencional. Los resultados del estudio dejaron en evidencia el interés de los estudiantes frente al conocimiento de las personas creadoras, el deseo de iniciar un proceso escritural de composiciones de los diversos subgéneros, el registro y publicación de las creaciones de Santa Ana-Magdalena, el uso didáctico en medios de comunicación y entornos virtuales.

Palabras clave: investigación como estrategia pedagógica, investigación, género lírico.

\section{Abstract}

Research as a pedagogical strategy (IEP) is an educational methodology that allows the integration of research processes into the classroom, in this study it will contribute to the knowledge of the lyrical genre contributing to the development of skills such as reading comprehension and students' superior cognitive processes. The purpose of the study is to implement research as a pedagogical strategy for the development of reading skills through the lyrical genre. The methodology was oriented under the qualitative approach, using the type of action research, from a descriptive, exploratory design. For the collection of data, techniques such as; documentary analysis The population was conformed by fifty (50) students of the IED Antonio Bruges Carmona of the Municipality of Santa Ana Magdalena, for this a non-probabilistic sampling of intentional type was realized. The results of the study showed the students' interest in the knowledge of the creative people, the desire to initiate a scriptural process of compositions of the various subgenres, the registration and publication of the creations of Santa Ana-Magdalena, the didactic use in media and virtual environments.

Keywords: research as a pedagogical strategy, research, lyrical genre.

1 Especialistas de la IED Antonio Bruges Carmona, sede Principal, pertenecientes al grupo de investigación Huellas Vernáculas.

- The author; licensee Universidad de la Costa - CUC. 


\section{Introducción}

La historia de la poesía en Colombia es un terreno que no se ha consolidado todavía en la investigación pedagógica, aunque se pueden constatar algunos avances sobre este tema, no solo en el conocimiento mismo de él, sino en algunas prácticas investigativas. Muchos trabajos en este terreno exigen una revisión detallada por la cantidad de interrogantes con relación al progreso de esta disciplina en otros países de América Latina. Colombia recientemente ha dado evidencias sobre aportes importantes a su historia, antropología y sociología. Siendo la literatura popular la menos afectada como Freija (2010).

Es necesario recalcar que, en Colombia se halla retardada la labor que, como la civilización en ese sentido, le ha correspondido llevar su parte a una verdad en pertinencia a un acervo común de la especie, formado por esa ciencia que Grimm, Muller, Bopp y tantos otros sabios han glorificado, buscando en las tradiciones de cada folklore regional el lazo que emparenta las varias religiones y lenguas de los hombres. Desde el punto de vista del folklore hay un interés general de estas experiencias poéticas, como parte de la acumulación de experiencias vivenciales de muchos años, juntando, analizando y comparando textos de gran valor literario, musical, etnográfico y antropológico de este conjunto de creencias, costumbres, ritos, artes y tecnologías transmitidas tradicionalmente a la sociedad.

Aunque para la transmisión de estas manifestaciones artísticas se ha llevado a la omisión de cierta cantidad de literatura que solo se ha transmitido de forma oral, por lo cual no aparece en la historia de la literatura colombiana. Dominando la poesía escrita a la oral desde la constitución misma de las ciudades y relegándola a un espacio cultural en ciertos pueblos de algunas localidades de regiones apar- tadas como resultado de una hegemonía cultural.

De acuerdo con Rama (2006):

La constitución de la literatura, como un discurso sobre la formación, composición y definición de la nación, habría de permitir la incorporación de múltiples materiales ajenos al circuito anterior de las bellas letras que emanaban de las élites cultas, pero implicaba asimismo una previa homogenización e higienización del campo, el cual sólo podía realizar la escritura (p. 9).

En el municipio de Santa Ana - Magdalena, no existe una compilación de las creaciones locales del género lírico, así mismo no se han trazado planes o programas desde la administración municipal y su ente rector, la casa de la cultura, para la motivación de la producción de este subgénero literario. Por otra parte, en los diseños curriculares de los proyectos educativos institucionales de las instituciones educativas existentes, no existen planes de estudios encaminados a potenciar y cultivar la competencia literaria que trascienda en composiciones de este tipo.

Esta realidad obedece a la falta de seguimiento en los programas de gobierno municipal, si se tiene en cuenta que, en una administración, donde se ha pretendido organizar eventos que fortalezcan esta dimensión poética, no ha tenido continuidad en el subsiguiente, además al cumplimiento irrestricto de los lineamientos y estándares curriculares, los cuales son asumidos por los educadores como una secuencia inalterable, a fin de darle lo cumplimiento a lo normado desde el Ministerio de Educación, y a los contenidos exigidos en la evaluaciones externas.

No obstante, a través de presentaciones en actos culturales se perciben personas con habilidades para la composición de versos, coplas, poemas y canciones 
populares, donde el amor, la soledad, la muerte, la contemplación del paisaje, los recuerdos, entre otros temas son tratados con especial subjetividad, relevante expresividad y finalidad estética. Y es de elevada connotación el número de niños y jóvenes que demuestran en jornadas culturales escolares y encuentros cívicoculturales municipales sus destrezas de hablantes líricos.

Así mismo se aprecian a adultos expresar con admirable talento sus idearios, emociones y sentimientos, a través de su voz poética en recitales, y en la Serenata a Santa Ana, magno certamen realizado anualmente en el marco de las Fiestas Patronales. Actividades culturales que permiten a los habitantes de la población el goce por los cantautores que con reconocida destreza abren su mundo interno para exaltar la exuberancia paisajística del territorio, las costumbres tradicionales, las añoranzas del pasado, la Patrona Santa Ana, abuela de Jesucristo, normas sociales pasadas, la cultura ribereña del Brazo de Mompox y facilitan a los moradores el encuentro con elementos culturales ancestrales. Según Menendez (1973, citado por Freja, 2010):

Es necesario recalcar que, las admiraciones colectivas a sus creaciones artísticas son poco conocidas, en virtud de ser un acontecimiento que surge en un proceso de regocijo popular anual, cuya organización ocurre previa al evento cultural con limitada planeación y nada de divulgación a nivel nacional, y por no existir mecanismos, para un proceso de compilación sistemática de estas creaciones liricas, y de devolución a la comunidad de tales constructos culturale (2010)s.

Definiendo la idea de popular como la conformación de una cultura uniforme, donde existe una lengua única en torno a diferentes formas culturales válidas y reconocidas, como afirma Freija, (2010):
Es necesario anotar que dada la subvaloración de estos personajes y de sus creaciones literarias, las cuales en muchas oportunidades son motivo de ridiculización e indiferencia por parte de la comunidad al considerarlas de poca relevancia e intelectualidad, no existe un registro que permita su conocimiento, divulgación y perpetuidad, y lo poco que se conoce de su obra ha sido por transmisión meramente oral. Lo anterior, conduce a determinar como efecto de lo anterior, la falta de continuidad en la orientación, estimulación, reconocimiento de estas creaciones líricas, el desconocimiento de estas por toda la comunidad, la desmotivación de los creadores, y la poca percepción de su existencia en el espectro municipal, nacional y global. Siendo estas poco reconocidas y relegadas en un tipo de clasificación literaria que va desde lo culto a lo popular (2010).

En este de orden de ideas, se considera de absoluta significación estas construcciones, por cuanto los temas abordados dan cuenta de la valoración por las costumbres folcloricas, la belleza paisajista, los grandes amores, reminiscencia de la infancia, la femineidad, vivencias de personajes destacados, situaciones límites familiares que exaltan la identidad cultural local. Por otra parte, son conocidas las expresiones líricas como las décimas, los pregones, los cantos de vaquería, las canciones de cuna, que testimonian el aprecio por las actividades económicas, hábitos, normas sociales y usos cotidianos del campesino, de la ama de casa, del trabajador rural.

Estas expresiones de poesía oral son comparables a las obras homéricas del mismo valor de La Ilíada y la Odisea por encontrarse en la pureza del pueblo y ser parte de la tradición verbal de este, transmitiendose como parte del folklor y de las tradiciones populares de la espontaneidad de sus habitantes. Menéndez (1953, citado por Freja, 2010) hace especial exhaltación de esto al señalar que: 
Es necesario recalcar que, en la actualidad, existen propuestas metodológicas de enfoques basados en investigación, entendidos como la forma de materializar lo que se hace y como se implementa donde surgen infinidad de propuestas metodológicas que se basan en un enfoque o toman varios para hacer de ellos una propuesta metodológica; es el caso de las pedagogías basadas o fundadas en investigación, las cuales se desarrollan en diferentes contextos y asumen variados caminos en coherencia con los paradigmas y corrientes en los cuales se inscriben. Dentro de esta teoría basada en investigación se encuentra la Enseñanza por descubrimiento y el Programa Ondas, con su propuesta de la Investigación como Estrategia Pedagógica (IEP), como una perspectiva que se desarrolla en ese enfoque, toma elementos de la pedagogía critico-liberadora de un enfoque socio-cultural (Mejía y Manjarrés, 2010).

Mediante la estructura del curricular los contenidos de un proceso educativo se convierten en cierto modo en un problema, y a partir de preguntas estructuradas, los avances se desarrollan de los estudiantes, determinando un lenguaje, raciocinios y conocimiento, Este concepto no es necesariamente autónomo, por el contrario, se hace guiado por el docente, quien se encarga de planificar los ejercicios y los procedimientos pertinente para tal fin (Pozo, 2006).

En ese sentido, existe un segundo hecho, que va enfocado en el reconocimiento en el lugar de trabajo de un material inmaterial, trabajo intelectual, que ha tomado en la sociedad actual, al convertirse en un generador de valor y, por lo tanto, elemento central para construir el capitalismo de este tiempo, lo cual convierte a actores que trabajan con el conocimiento, la tecnología, la información y la comunicación en sujetos claves de los nuevos procesos de conformación de esta sociedad, alcanzando los educadores de todos los tipos un carácter que organiza y da forma a base y fundamento de estas modificaciones sociales (Mejía, 2011).
Hay que mencionar, además que, la investigación como estrategia pedagógica, busca postular la existencia de saberes que se enfocan más en la experiencia y las situaciones de la sociedad en pertinencia mediante conocimientos que están dados con base a unos conocimientos que están más dados en la esfera de las disciplinas; de esta forma, postula la existencia de racionalidades diferentes (Mignolo, 2003).

Por sobre todo, las cuales también deben ser reconocidas por el ejercicio educativo, haciendo real de una forma participativa, basada en la interculturalidad y la negociación cultural; que , además debe darle cabida a las razones más allá de la "razón universal", para hacer visible el reconocimiento del otro y de lo otro diferente a aquello que es postulado como universal, fruto de la lógica de control y de poder, visibilizar en los diferentes saberes propios de una sociedad cada día mas urgida en ese sentido, (De Souza, 2001), en el respeto y el reconocimiento de los aportes de cada uno. Por ello en la IEP, maestro y maestra se convierten en acompañantes co-investigadores, transformando su rol tradicional y reconociéndose como aprendientes en el proceso.

Por su parte, la investigación como estratégica pedagógica, en la mayoría de la veces se parte problema según el sentido común del estudiante, esto con la finalidad de elaborar con base a perspectivas en la educación popular, que reconoce la existencia de saberes comunes y elaborados así en la negociación con los conocimientos disciplinares, y de las maneras como se correlacionan en la negociación cultural (Mariño, 2010), lo cual muestra la manera en que se van dando estas transformaciones, a través del aprendizaje colaborativo, en el cual intervienen niños y adultos. En el sentido de Vygotsky, toda función aparece en dos planos: en lo social terpsíquico) y en lo psicológico (intrapsíquico). 
Así entonces, las organizaciones, señalan Mendoza, López y Salas (2016), "son entes que generan sus propios objetivos y diseñan la forma como alcanzarlos teniendo en cuenta, necesariamente, las influencias del entorno social" (p. 62). Las escuelas no escapan de ello y es de allí que, en las organizaciones cualquiera que sea su ámbito, se hace preciso introducir una gestión estratégica como una preocupación por posicionarse. (Mendoza, López, \& Salas, 2016)

En el entendido de la sociedad, exponen Hernández y Jiménez (2015), "no reclama sólo la ética individual de las personas que componen las organizaciones, sino que ven un ente con responsabilidad propia económica, social y medioambiental a la que debe exigírsele comportamientos acordes a la misma" (p. 11) según su razón de ser. Con lo cual "se podrá avanzar en el conocimiento sobre las condiciones de creación y de permanencia, además del dinamismo del sistema social".(Maculan, Jiménez y Castellanos, 2015, p. 16)

\section{Metodología}

Se realizó un estudio de tipo cualitativo, utilizando el modelo de investigación acción, desde un diseño descriptivo, exploratorio. La investigación cualitativa busca conocer e interpretar la realidad de los participantes a través de sus propias experiencias, entregando una información subjetiva del fenómeno de estudio. (Hernández, Fernández, y Baptista, 2010). Por su parte el modelo de investigación acción plantea una producción de conocimiento basado en la reflexión de los participantes, teniendo en cuenta la participación activa de maestros en el proceso de enseñanza y acompañamiento tecnológico, guiando al estudiante en su proceso de investigación. Generando nuevo saber y conocimiento sobre una realidad determinada en un proceso de aprendizaje colaborativo.
Los estudios con un diseño descriptivo están encaminados a especificar las características de la población sujeto de estudio o los fenómenos que sean sometidos a análisis. Bajo un estudio de tipo exploratorio, debido a que no se han realizado estudios previos en la institución. (Hernández, Fernández, y Baptista, 2010). La población estuvo conformada por cincuenta (50) estudiantes de la IED Antonio Bruges Carmona del Municipio de Santa Ana Magdalena, para esto se realizó un muestreo no probabilístico de tipo intencional utilizando como criterio de inclusión el interés artístico y musical.

También se realizó una recolección de información a través de entrevistas semiestructuradas e historias de la vida de las personas creadoras de las creaciones literarias del género en mención representada por los poetas, compositores y copleros del municipio de Santa de Ana de Buenaventura, las cuales fueron subidas online a diversas plataformas como YouTube, Facebook y Twitter.

\section{Resultados}

A partir de los instrumentos de recolección de datos, se logró interpretar la realidad con un horizonte subjetivo frente a las representaciones sociales y/o culturales del genero lirico del Municipio de Santa Ana, lo anterior se presenta en lo que concierne a como surgen las creaciones liricas, se encontró en los argumentos de los entrevistados, que se daba a partir del deseo y del gusto por componer y escribir, además la imitación a padres, a abuelos, la lectura de versos de poetas reconocidos.

En lo referente al tema motivo de inspiración y/o contenido de los canticos, se encontró que circundan a paisajes, amores prohibidos, costumbres de la época y añoranza del pasado, de manera que, en el contexto y/o entorno que rodea las composiciones generalmente se desarrollaron 
aislados de la noche, en la contemplación de la naturaleza y/o en un momento de soledad. Con la experiencia vivida en torno a sus composiciones los entrevistados señalaron, que han vivenciado diversas emociones, tales como tristeza evocando el pasado, juegos infantiles, costumbres pasadas, sus antecesores, su vida familiar, pautas, usos y costumbres en su hogar, entre otros.

En virtud de la necesidad, es decir al seguir interrogando que se requiere para componer han señalado, la disposición, un ambiente propicio de tranquilidad, y la mayoría anota que es innato, pues creen que su competencia escritural poética, ha surgido de su parte genética. Referido al reconocimiento y el éxito por sus composiciones, expresan en un reducido número que ello ha sido verbigracia viajes al territorio colombiano, y a países extranjeros para divulgar sus creaciones, otros han asegurado que escasamente las publican en un evento municipal donde se rinde culto anualmente en las festividades patronales, y la gran mayoría anotan que las dan a conocer en actos culturales de instituciones educativas a los cuales son invitados.

Se registraron y publicaron las creaciones del género lírico de los compositores de Santa Ana-Magdalena a fin de facilitar su preservación, conocimiento y difusión en las actuales y futuras generaciones mediante el uso didáctico de los medios de comunicación, uso de entornos virtuales de enseñanza, creación y análisis de materiales multimediales e hipertextos tales como la emisora local, canales de propagación virtual (YouTube, Facebook, Twitter,) y consolidación de la compilación en redes sociales y líneas temáticas análogas al proyecto, con la consideración que para poder llevar a cabo con éxito innovaciones educativas, estas deben ser gestadas desde una cultura escolar participativa, dinámica, creativa, donde el educando haga ruptura de ser un simple ejecutor.
Por su parte, siendo el principal medio de propagación de los resultados de este proyecto de investigación el espacio "El maestro tiene la palabra" en donde se expuso ante otros educadores los alcances y nivel del presente proyecto de investigación. Se hizo un redimensionamiento del diseño curricular, mediante la orientación de la asignatura lenguaje y cultura local, constructo transversal de las áreas Ciencias Sociales, Humanidades y Lengua Castellana, Educación Ética y Valores, Educación Artística y Cultura, Ciencias Naturales y Educación Ambiental, que se implementan en la Institución Educativa Antonio Bruges Carmona, a partir del impacto generado en los educandos, y la motivación por ser creadores de obras similares.

Al ser la institución educativa departamental el escenario favorable para propiciar la creación textual, no existen planes curriculares, que propendan por la construcción de estos subgéneros literarios y la valoración de las existentes, ello en razón de la orientación absoluto del cumplimiento de estándares nacionales. En virtud de lo planteado, de no diseñarse un trabajo que permita el conocimiento, la compilación y divulgación de estas obras, que expresan generalmente la sabiduría popular del santanero, la generación presenta desconocerá el amplio sentir y pensar a través de la lírica, y por ende valorará superlativamente universos poéticos de otras latitudes, que conducirán en el detrimento del aprecio por su idiosincrasia, y al desinterés por su construcción en el escenario favorable de las instituciones educativas.

\section{Conclusiones}

A partir de lo referido y desde la mirada interdisciplinar que se ha dado a la presente investigación, se han logrado resultados que se relacionan con el empoderamiento de los estudiantes frente a conocer el género lirico del Municipio de Santa Ana y de quie- 
nes han sido los impulsadores de este. Por otra parte, se registra y publica las creaciones del género lírico de los compositores de Santa Ana-Magdalena a fin de facilitar su preservación, conocimiento y difusión en las actuales y futuras generaciones mediante el uso didáctico de los medios de comunicación, uso de entornos virtuales de enseñanza, creación y análisis de materiales multimediales e hipertextos tales como la emisora local, canales de propagación virtual (YouTube, Facebook, Twitter,) y consolidación de la compilación en redes y líneas temáticas análogas al proyecto,

Para poder llevar a cabo con éxito innovaciones educativas, estas deben ser gestadas desde una cultura escolar participativa, dinámica, creativa, donde el educando haga ruptura de ser un simple ejecutor. El proceso de rediseño curricular en la institución hacia la contextualización de este proyecto hizo evidente la imperiosa necesidad de hacer de la articulación: práctica pedagógica e investigación, específicamente en la función y en la coherencia requerida en el momento histórico actual de dos procesos pedagógicos con identidad propia: investigación y didáctica Constituyo una experiencia de regocijo y deleite de la indagación, reflexión y consenso permanente sobre inquietudes, hallazgos, descubrimientos, construcción de saberes que involucran procesos de investigación en el desarrollo curricular.

Este trabajo constituye el desafío de ser los docentes diseñadores, gestores, animadores y asesores de experiencias de aprendizajes que no estén condicionados por horarios, distancias o por limitaciones personales, apostándole a propiciar diversos sistemas simbólicos, a orientar el pensar estratégicamente, y el aprender en forma permanentemente, desde el redimensionamiento del diseño curricular, mediante la orientación de la asignatura lenguaje y cultura local, constructo transversal de las áreas Ciencias Sociales, Humanidades y
Lengua Castellana, Educación Ética y Valores, Educación Artística y Cultura, Ciencias Naturales y Educación Ambiental, que se implementa en la Institución Educativa Antonio Bruges Carmona, a partir del impacto generado en los educandos, y la motivación por ser creadores de obras similares.

\section{Referencias}

Ausubel, D. (2001). Teoría del aprendizaje significativo. Fascículos de CEIF, 1. 1-10.

Cochran, M. y Lytle, S. (2003). Más allá de la certidumbre: Adoptar una actitud indagadora sobre la práctica. En, A. M. Liberman y J. L. Miller (Eds.), La indagación como base de la formación del profesorado y la mejora de la educación. Barcelona: Octaedro.

De souza, J. F. (2001). Atualidade de Paulo Freire. Contribuição ao debate sobre a educação na diversidade cultural. Recife: Edições Bagaço.

Edwards, D. y Mercer, H. (1987). El conocimiento compartido: El desarrollo de la comprensión en el aula. Barcelona: Paidós-MEC.

Freja, A. (2012). Romances, coplas y décimas en el Pacífico y el Caribe colombiano: poética de una literatura oral en Colombia. [Tesis]. Universidad Nacional de Colombia. Bogotá, D.C.

Hernández, R., Fernández, C. y Baptista, P. (2010). Metodología de la investigación. México, D.F.: Editorial Mc Graw Hill.

Hernández, H. y Jiménez, A. (2015). Responsabilidad social empresarial, zona urbana del distrito de Barranquilla: estudio etnográfico. Económicas CUC, 36(2), 9-18.

Maculan, A-M., Jiménez, C. y Castellanos, O. (2015). Aprendizaje en el proceso de incubación de empresas de base tecnológica. Económicas CUC, 36(1), 15-48. 
Mandón, S. (2016). Las Lecturas narrativas infantiles para mejorar el nivel de comprensión lectora en los grados de escuela nueva de la Institución Educativa Edmundo Velásquez a través de las TIC. Ocaña - Norte de Santander

Mejía, M. y Manjarrés M. (2010). Las pedagogías fundadas en la investigación. Búsquedas en la reconfiguración de la educación. +Revista Internacional Magisterio, 42. 16-26.

Mejía, M. (2011). Las escuelas de la globalización. El conflicto por su reconfiguración. Bogotá, D.C.: Desde Abajo.

Mendoza, D., López, D. y Salas, E. (2016). Planificación estratégica de recursos humanos: efectiva forma de identificar necesidades de personal. Económicas CUC, 37(1), 61-79.

Menéndez, R. (1953). Romancero hispánico (hispano-portugués, americano y sefardi) teoría e historia. Madrid: Espasa-Calpe.

Mignolo, W. (2003). Capitalismo y geopolítica del conocimiento. Buenos Aires: Paidós.
Núñez, E., Vergara, R. y Bocanegra, J. (2014). Sistema experto basado en lógica difusa tipo 1 para determinar el grado de riesgo de preeclampsia. INGE CUC, 10(1), 43-50. Recuperado de https://revistascientificas.cuc.edu.co/ ingecuc/article/view/341

Otero, G. (1928). La literatura colonial de Colombia. La Paz: Imprenta Artística.

Pozo, J. y. (2006). Aprender y enseñar ciencia. Madrid: Morata.

Rama, Á. (2006). Crítica literaria y utopía en América Latina. Medellín: Editorial Universidad de Antioquia.

Schön, D. (1991). The Reflective Practitioner. Aldershot: Ashgate Publishing Ltd.

Vergara, J. (1974). Historia de la literatura en la Nueva Granada. Vol. III. Bogotá, D.C.: Biblioteca Banco Popular. 